\title{
ST Elevation Myocardial Infarction due to Cocaine Induced Coronary Vasospasm
}

Ibrahim Kassas*, Craig S. Smith and Jeffrey Shih

Division of Cardiology, University of Massachusetts Medical School, Worcester, MA, USA

*Corresponding author: Ibrahim Kassas, Division of Cardiology, University of Massachusetts Medical School, Worcester, MA, USA, E-mail: ibrahim.kassas@umassmemorial.org

Received date: September 24, 2015; Accepted date: September 28, 2015; Published date: September 30, 2015

Copyright: (c) 2015 Kassas I, et al. This is an open-access article distributed under the terms of the Creative Commons Attribution License, which permits unrestricted use, distribution, and reproduction in any medium, provided the original author and source are credited.

\section{Case Study}

A 59-year-old man with medication-treated hypertension, noninsulin dependent diabetes mellitus and a current tobacco user, presented with substernal pressure-like chest pain of one hour duration, prior to arrival to the emergency room. Electrocardiography on arrival (Figure 1A) showed anterior ST segment elevations with subsequent anterior T-wave inversions. Initial troponin concentration was $72.58 \mathrm{ng} / \mathrm{mL}$ (reference range: $0.01-0.04 \mathrm{ng} / \mathrm{mL}$ ). He underwent emergent coronary angiography, which revealed $50 \%$ mid-left anterior descending (LAD) artery stenosis with severe vasospasm involving the entire LAD segment with TIMI 1 flow down the artery (Figure 1B). Intra-coronary nitroglycerin administration resolved the vasospasm and TIMI 3 flow was restored (Figure 1C). Subsequent echocardiography showed a depressed left ventricular ejection fraction to $40 \%$ with wall motion abnormalities consistent with a LAD territory infarct. Urine toxicology screen showed evidence of cocaine and the patient eventually admitted to using cocaine prior to the onset of the chest pain. He was eventually discharged after being medicated with calcium channel blockers and nitrates. He was also started on carvedilol, aspirin, atorvastatin, lisinopril and spironolactone for management of his ischemic cardiomyopathy and concomitant atherosclerosis. Prior evidence suggests the safety of non-selective beta-blockade in the setting of cocaine use which was demonstrated in our case [1-4]. The patient was counseled extensively about abstinence from illegal drugs.

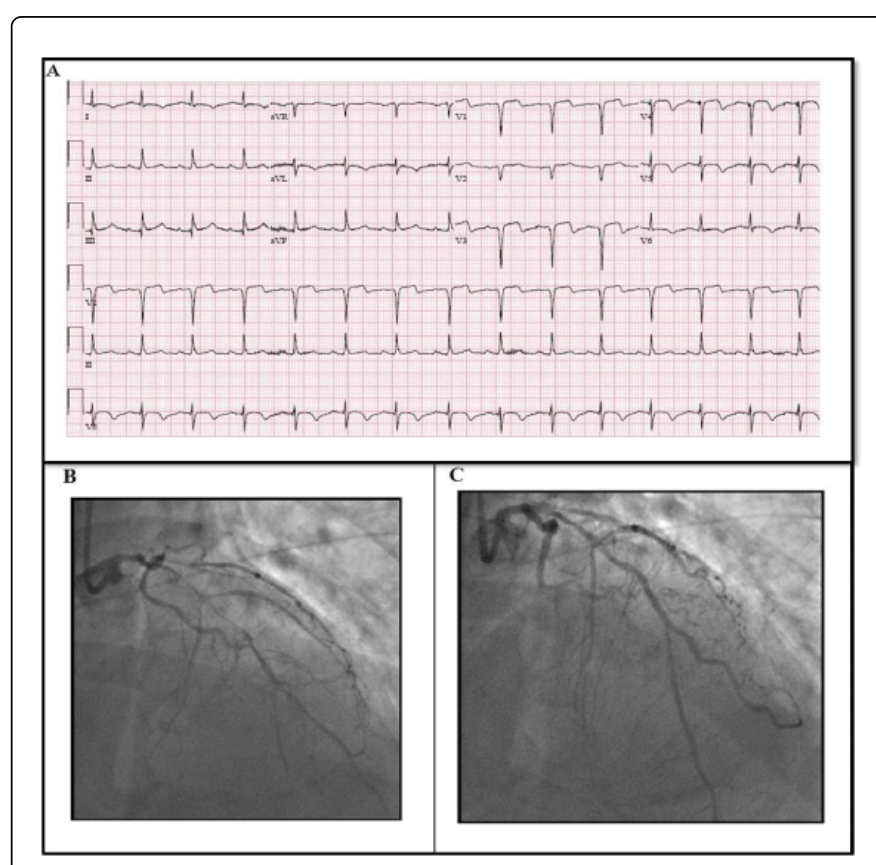

Figure 1: (A) Echocardiography on arrival; (B) Emergent coronary angiography; (C) Restored vasospasm and TIMI 3 flow

\section{References}

1. Hoskins MH, Leleiko RM, Ramos JJ, Sola S, Caneer PM, et al. (2010) Effects of labetalol on hemodynamic parameters and soluble biomarkers of inflammation in acute coronary syndrome in patients with active cocaine use. J Cardiovasc Pharmacol Ther 15: 47-52.

2. Fanari Z, Ibrahim M, Hamilton A, Abdulhadi M, Laddu A, et al. (2012) Evaluation of the safety of beta-blockers in the acute management of cocaine-associated chest pain. J Am Coll Cardiol 59: E514.

3. Fanari Z, Kennedy KK, Lim MJ, Laddu AA, Stolker JM (2014) Comparison of in-hospital outcomes for beta-blocker use versus non-beta blocker use in patients presenting with cocaine-associated chest pain. Am J Cardiol 113: 1802-1806.

4. Lange RA, Hillis LD (2001) Cardiovascular complications of cocaine use. N Engl J Med 345: 351-358. 